\title{
Genetic Control of T Cell Receptor BJ Gene Expression in Peripheral Lymphocytes of Normal and Rheumatoid Arthritis Monozygotic Twins
}

Toshihiro Nanki, ${ }^{*}$ Hitoshi Kohsaka, ${ }^{\ddagger}$ Noboru Mizushima, ${ }^{*}$ William E.R. Ollier, ${ }^{\S}$ Dennis A. Carson,\| and Nobuyuki Miyasaka* ${ }^{*}$ First Department of Internal Medicine, ${ }^{\ddagger}$ Division of Immunological Diseases, Medical Research Institute, Tokyo Medical and Dental University, Tokyo, 113 Japan; ${ }^{\S}$ Arthritis and Rheumatism Council, Epidemiology Research Unit, University of Manchester, Manchester, M13QPT United Kingdom; and ${ }^{\|}$Department of Medicine and The Sam and Rose Stein Institute for Research on Aging, University of California, San Diego, La Jolla, California 92093

\begin{abstract}
The amino acids encoded at the junctions of $T$ cell receptor (TCR) $\mathrm{V}$ and $\mathrm{J}$ genes directly interact with MHC bound peptides. However, the regulation of the human TCRBJ gene repertoire has been difficult to analyze, because of the potentially complex number of $\mathrm{BJ}$ gene rearrangements. To overcome this problem, we developed a PCR-ELISA method to study BJ gene expression, and compared peripheral $\mathrm{T}$ lymphocytes from 12 pairs of monozygotic twins, including 6 rheumatoid arthritis (RA) discordant pairs, and 5 normals. Analyses of the TCRBV5, 13 and 17 gene families, which have been reported to be increased in RA patients, showed: (a) the three TCRBV transcripts have common features of BJ gene usage; (b) TCR transcripts from each TCRBV family display a distinctive BJ gene profile, which is displayed better by $\mathrm{CD}^{+}$than $\mathrm{CD}^{+}$lymphocytes; (c) the BJ gene repertoires of monozygotic twins are more similar than those of unrelated individuals; and (d) the inflammation of RA does not induce specific changes in the genetically determined pattern of BJ expression. These results indicate that the frequency of expression particular TCRBV-TCRBJ recombinants in human lymphocytes is controlled genetically, and is maintained despite the presence of a chronic inflammatory disease. (J. Clin. Invest. 1996. 98:1594-1601.) Key words: polymerase chain reaction - enzyme-linked immunosorbent assay $\cdot \mathrm{CD} 4$ positive T lymphocyte $\cdot \mathrm{CD} 8$ positive $\mathrm{T}$ lymphocyte $\cdot$ amino acid sequence
\end{abstract}

\section{Introduction}

The expressed T cell receptor (TCR) consists of a $\alpha$ and $\beta$, or $\gamma$ and $\delta$ chain heterodimer. More than $95 \%$ of peripheral T lymphocytes express TCR $\alpha \beta$ chains. The TCR variable regions of functional $\alpha$ and $\beta$ chains are constructed by rearrangement of variable (V), diversity (D) ( $\beta$ chain only), and joining (J) gene segments with additional noncoding $(\mathrm{N})$ regions $(2,3)$. Previ-

\footnotetext{
Address correspondence to Hitoshi Kohsaka, M.D., Division of Immunological Diseases, Medical Research Institute, Tokyo Medical and Dental University, 1-5-45, Yushima, Bunkyo-ku, Tokyo, 113 Japan. Phone: 81-3-5803-5819; FAX: 81-3-5684-0717; E-mail: kohsaka.imm@ tmd.ac.jp

Received for publication 7 May 1996 and accepted in revised form 3 July 1996.
}

J. Clin. Invest.

(C) The American Society for Clinical Investigation, Inc. 0021-9738/96/10/1594/08 \$2.00

Volume 98, Number 7, October 1996, 1594-1601 ous studies of the expressed TCRBV ${ }^{1}$ gene repertoires in peripheral blood lymphocytes (PBL) showed that they are controlled genetically (4), and primarily by HLA (5). These results may be explained by the direct interaction of the first and second complementarity determining regions (CDR1 and CDR2) of the TCR with MHC molecules during lymphocyte development. However, TCRBJ gene products are part of the third complementarity determining region (CDR3), ${ }^{2}$ which is thought to make contact with MHC bound peptides $(6,7)$. Thus, one might expect that the adult peripheral lymphocyte TCRBJ gene repertoire would be more dependent on environmental antigenic stimuli than the BV gene repertoire.

The regulation of human TCRBJ gene expression has not been extensively studied. In order to discern the relative importance of genetic and environmental effects on TCRBJ repertoire formation in outbred human populations, it is necessary to compare the expression of multiple different TCRBJ recombinants in a large number of related individuals. Previously, we developed a method to analyze the BV gene repertoire by PCR-ELISA (4). Here, we have modified this approach to study TCRBJ gene expression by PBL from 12 pairs of monozygotic twins, including 6 pairs of twins discordant for rheumatoid arthritis (RA). Because monozygotic twins share identical genetic backgrounds, differences in their TCRBJ repertoires must be due to environmental or stochastic factors. If a systemic autoimmune disease, such as RA, was triggered by a single potent environmental stimulus, the peripheral $\mathrm{T}$ lymphocyte TCRBJ repertoire might be skewed. In contrast, an antigenic peptide would not necessarily expand $\mathrm{T}$ cells with specific TCRBV gene products, that interact primarily with the MHC backbone.

Surprisingly, our results show that TCRBJ expression is controlled genetically. Even a chronic autoimmune syndrome such as RA has little influence on the overall pattern of TCRBJ expression.

\section{Methods}

Samples. Six pairs of healthy monozygotic twins (A1-A2, B1-B2, C1C2, D1-D2, E1-E2, F1-F2), six pairs of monozygotic twins discordant

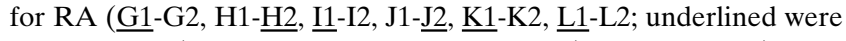
affected twins), and five healthy individuals (M, N, O, P, Q) were studied. Their ages ranged from $23-65 \mathrm{yr}$ (mean age: 37.7 ). Monozygosity was confirmed by PCR amplification of genomic DNA with

1. The TCR gene nomenclatures recommended by the World Health Organization-International Union of Immunological Societies Subcommittee are used in this paper (1).

2. Abbreviations used in this paper: $\mathrm{CDR} 3$, third complementary determining region; TMAC, tetramethylammonium chloride; VNTR, variable number of tandem repeats. 
Table I. Oligonucleotides Used for PCR-ELISA Assay

\begin{tabular}{ll}
\hline Name & \multicolumn{1}{c}{ Sequence } \\
\hline BV5 & CAG AGA AAC AAA GGA AAC TTC \\
BV13 & CCT GGT CGA \\
BV17s & CAA GGA GAA GTC CCC AAT \\
& TTT CAG AAA GGA GAT ATA GCT \\
BTN-BC1 & GAA GGG TAC \\
BTN-BC2 & biotin-TGT GGG AGA TCT CTG CTT C \\
& biotin-ACG CGT CGA CTT CTG ATG \\
BJ1.5c & GCT CAA ACA CA \\
BJ1.1p & TGG AGA GTC GAG TCC CAT CA \\
BJ1.2p & ACA AGG CAC CAG ACT CAC A \\
BJ1.3p & TTC GGG GAC CAG GTT AAC C \\
BJ1.4p & AGA GGG AAG TTG GCT CAC T \\
BJ1.5p & CAG TGG AAC CCA GCT CTC T \\
BJ1.6p & TGA TGG GAC TCG ACT CTC C \\
BJ2.1p & GAA TGG GAC CAG GCT CAC T \\
BJ2.2p & GCC AGG GAC ACG GCT CAC C \\
BJ2.3p & AGA AGG CTC TAG GCT GAC C \\
BJ2.4p & CCC AGG CAC CCG GCT GAC A \\
BJ2.5p & CGC CGG GAC CCG GCT CTC A \\
BJ2.6p & GCC AGG CAC GCG GCT CCT G \\
BJ2.7p & GGC CGG CAG CAG GCT GAC C \\
& GCC GGG CAC CAG GCT CAC G \\
\hline &
\end{tabular}

five pairs of oligonucleotide primers for variable number of tandem repeats (VNTR) (8) or by finger print analysis with anonymous probes. RA was diagnosed according to the American College of Rheumatology criteria (9). All patients were seropositive for rheumatoid factor and had active synovitis at the time when their blood samples were collected. HLA-DR haplotypes of RA patients were DR4/ 10 in G1-G2, DR4/9 in H1-H2, DR2 in I1-I2, DR2/11 in J1-J2, DR4 in $\mathrm{K} 1-\mathrm{K} 2$, and DR3/7 in L1-L2.

Oligonucleotides. The oligonucleotides used for the PCRELISA assay are shown in Table I. BV5s, 13s, and 17s were TCRBV5, 13, and 17 specific sense PCR primers, respectively. BTN$\mathrm{BC} 1$ was a TCRBC gene-specific anti-sense PCR primer. BTN-BC2 was used to generate a standard curve for ELISA. BTN-BC1 and BTN-BC2 were labeled with biotinylated phosphoramadite (Clontech, Palo Alto, CA) at their 5' ends. BJ1.5c was a TCRBJ1S5-specific anti-sense primer used to amplify junctional regions of TCRBV17S1J1S5 transcripts.

The TCRBJ specific oligonucleotides (BJ1.1p-BJ2.7p) used for hybridization are shown in Table I. During the rearrangement of BD-BJ gene segments, BJ genes are truncated to variable lengths at the $5^{\prime}$ ends. We analyzed 268 rearranged TCRB transcripts which were published previously or cloned in our previous studies, to determine the maximal length of the deletion. All BJ probes specified 3' parts of BJ genes which were never truncated by rearrangement.

Separation of cells. PBL from one pair of healthy monozygotic twins (B1-B2) and two pairs discordant for RA (G1-G2, H1-H2) were separated into $\mathrm{CD}^{+}$and $\mathrm{CD} 8^{+} \mathrm{T}$ cell subsets. 5-10 $\times 10^{6} \mathrm{PBL}$ were stained with FITC-conjugated anti-CD4 monoclonal antibody (mAb) (T4; Coulter, Miami, FL) and PE-conjugated anti-CD8 mAb (T8; Coulter). $\mathrm{CD}^{+}$and $\mathrm{CD}^{+} \mathrm{T}$ cell subsets were separated using a fluorescence activated cell sorter (EPICS Elite Flow Cytometer; Coulter). Purity of the separated cells was estimated to be $>97 \%$.

$c D N A$ preparation and PCR. PBL were separated by FicollHypaque gradient centrifugation. Total RNA was prepared from total PBL, sorted $\mathrm{CD}^{+}$and $\mathrm{CD} 8^{+} \mathrm{T}$ cells, using TRIzol (Life Technologies Inc., Gaithersburg, MD). First strand cDNA was synthesized using oligo-dT primers and Superscript II reverse transcriptase (Life Technologies Inc.). $10 \mathrm{ng}$ cDNA was amplified by PCR in $100 \mu \mathrm{l}$ of 10
$\mathrm{mM}$ Tris- $\mathrm{HCl}(\mathrm{pH} 8.3), 50 \mathrm{mM} \mathrm{KCl}, 1.5 \mathrm{mM} \mathrm{MgCl}, 0.001 \%$ gelatin, $200 \mu \mathrm{M}$ dNTP, and 5 U Taq DNA polymerase (Boehringer-Mannheim Biochemicals, Indianapolis, IN). The PCR was performed with BV specific primers (BV5s, BV13s, or BV17s) and BTN-BC1. The cycling program was: $94^{\circ} \mathrm{C}$ for $1 \mathrm{~min}, 60^{\circ} \mathrm{C}$ for $2 \mathrm{~min}$, and $72^{\circ} \mathrm{C}$ for $3 \mathrm{~min}$ for 35 cycles, followed by a final extension for $10 \mathrm{~min}$. The PCR products were purified using mini-columns (Wizard PCR Preps; Promega Corp., Madison, WI).

For testing cross-hybridization of the $13 \mathrm{BJ}$-gene-specific probes, we used 13 different TCR clones, each of which contained a different $\mathrm{BJ}$ recombination. These clones were ligated with pT7Blue T-Vector (Novagen, Inc., Madison, WI). The BJ gene regions were amplified with corresponding BV gene specific primers (10) and BTN-BC1.

To compare labeling and hybridization efficiencies of the probes, the three nucleotide sequences recognized by BJ1.1p, BJ1.2p, and $\mathrm{BJ} 1.4 \mathrm{p}$ were arbitrarily selected, and tandemly inserted into the multi-cloning site of pBluescript II SK + (Stratagene Inc., La Jolla, CA). The insert was PCR amplified with a biotinylated T7 primer and a T3 primer, and used for hybridization with the labeled probes.

Analysis of TCRBJ gene repertoires. The method to analyze TCRBJ gene repertoires was adapted from the PCR-ELISA technique used to analyze BV genes (4). The PCR products were immobilized onto streptavidin-coated 96 well ELISA plates. The BJ-specific probes were labeled with digoxigenin at $3^{\prime}$ ends and placed into the wells. Three molar tetramethylammonium chloride (TMAC) was added to the hybridization solution $(150 \mu \mathrm{l}$ of $5 \mathrm{nM}$ solution of each digoxigenin labeled sense-strand oligonucleotide probe, $0.1 \mathrm{M}$ sodium phosphate buffer [pH 6.8], 1 mM EDTA, 5X Denhardt's solu-
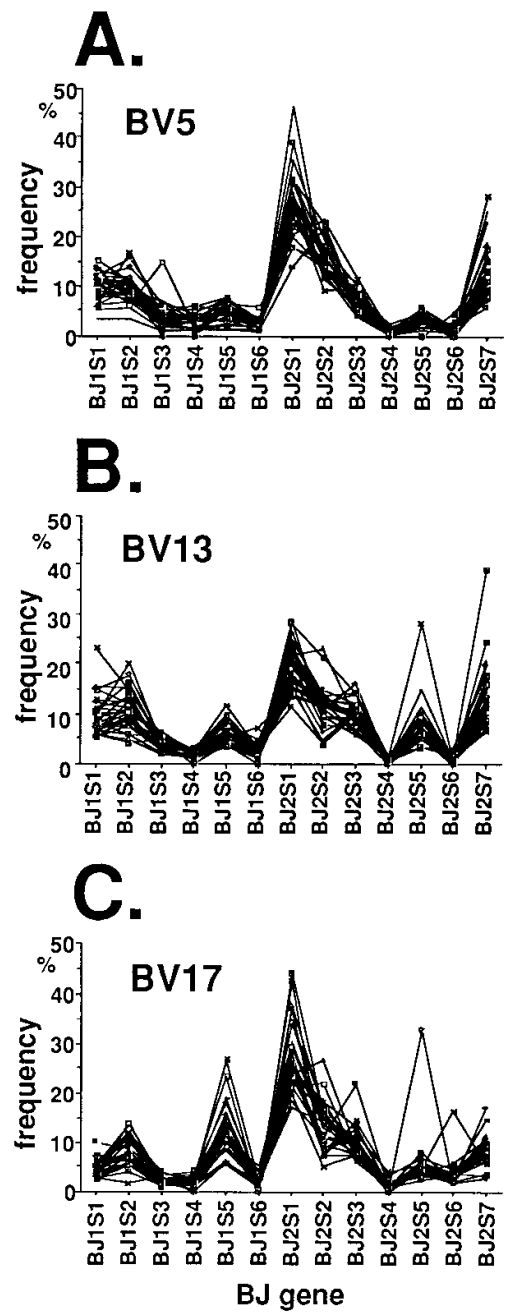

Figure 1. TCRBJ gene frequencies of peripheral T lymphocytes from six pairs of healthy monozygotic twins, six pairs of monozygotic twins discordant for $\mathrm{RA}$, and five normal individuals. (A) BJ gene repertoire of TCRBV5 transcripts. $(B) \mathrm{BJ}$ gene repertoire of BV13 transcripts. $(C) \mathrm{BJ}$ gene repertoire of BV17 transcripts. 


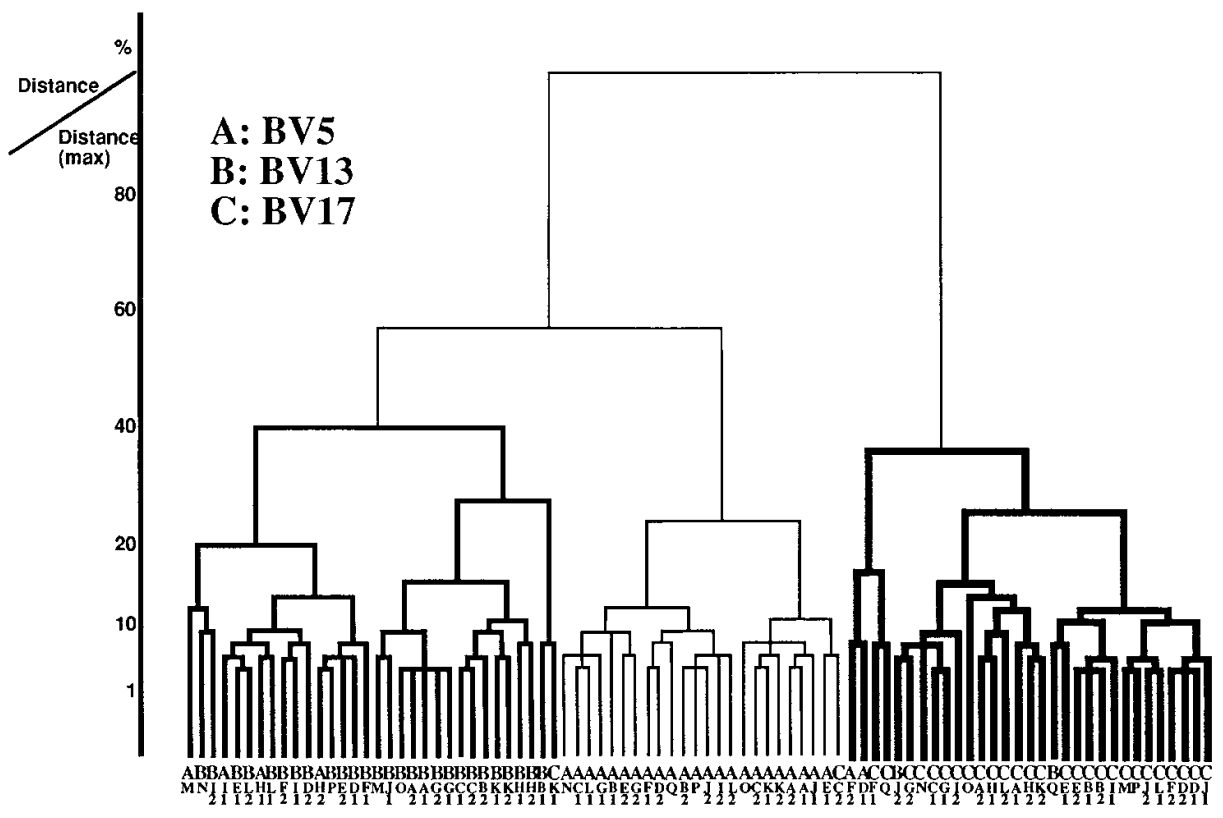

Figure 2. Cluster analysis of TCRBJ gene repertoires. From all BJ gene repertoire data, the distance of every combination of two BJ gene repertoires was calculated by Ward's method and Cityblock (Manhattan) distance. All calculated distances, divided by the maximum distance, are shown as a dendrogram. (A) BJ gene repertoires of TCRBV5 transcripts. (B) BJ repertoires of BV 13 transcripts. $(C) \mathrm{BJ}$ repertoires of $\mathrm{BV} 17$ transcripts. Listed below are the identifications of the donors. tion, and $0.6 \%$ SDS). In this buffer, the dissociation temperature of each BJ specific probe depends mainly on length and not on nucleotide composition (11). All probes had the same length so that the use of TMAC ensures equal hybridization efficiency of the 13 different probes. The hybridization was at $52^{\circ} \mathrm{C}$ for $90 \mathrm{~min}$. The plates were washed once with warmed $\left(52^{\circ} \mathrm{C}\right)$ TMAC wash solution $(3.0 \mathrm{M}$ TMAC, $50 \mathrm{mM}$ Tris- $\mathrm{HCl}[\mathrm{pH} 8.0]$, and $0.2 \%$ SDS) and twice with $6 \mathrm{X}$ SSC and $0.1 \%$ sodium $N$-lauroyl sarcosinate.

After the wells were washed, the plates were incubated with antidigoxigenin antibodies labeled with peroxidase (Boehringer-Mannheim), and reacted with tetramethylbenzidine microwell peroxidase substrate (Kirkegaard \& Perry Laboratories, Inc., Gaithersburg, MD). The absorbance values were recorded at $450 \mathrm{~nm}$ with a microplate reader (Molecular Dynamics, Menlo Park, CA). For generating a standard curve, serial dilutions of BTN-BC2 labeled with digoxigenin at the $3^{\prime}$ end was applied onto the plates, and treated the same way. According to the standard curve, the absorbance values were transformed to concentrations with Deltasoft II software (Biometallics, Inc., Princeton, NJ). The frequency of each BJ gene usage was calculated by dividing the concentration of each $\mathrm{BJ}$ gene by the total concentration of BJ genes.

Analysis of junctional region sequences. Junctional regions of TCRBV17S1J1S5 transcripts were amplified from PBL cDNA of one pair of healthy monozygotic twins and one healthy individual, using $\mathrm{BV} 17 \mathrm{~s}$ and $\mathrm{BJ} 1.5 \mathrm{c}$ primers. The amplification conditions were the same as for the BJ gene repertoire analysis. The PCR products were purified with minicolumns and directly ligated with pT7Blue T-Vector. The TCR junctional region sequences of the recombinant clones were determined with an automated sequencer (model 373A; Applied Biosystems, Inc., Foster City, CA).

Statistical analyses. Multivariate cluster analyses of the TCRBJ gene repertoire data were performed by Ward's method, including
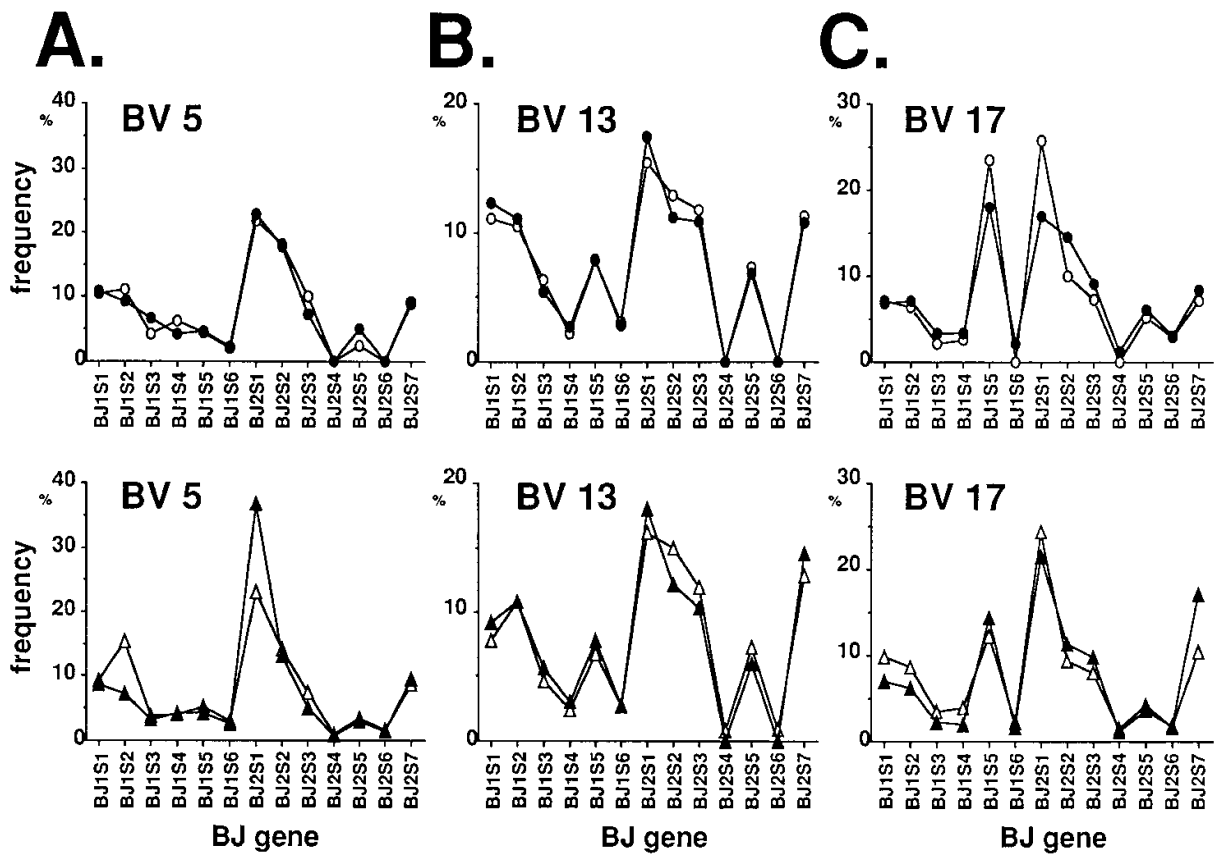

Figure 3. Representative TCRBJ gene repertoires of PBL from monozygotic twins. (A) BJ gene repertoires of TCRBV5 transcripts of two pairs of monozygotic twins. $(B) \mathrm{BJ}$ repertoire of BV13 transcripts. $(C) \mathrm{BJ}$ repertoires of BV17 transcripts. A1 $(O)$ and A2 (•) are healthy monozygotic twins. G1 $(\triangle)$ is RA affected and G2 $(\Delta)$ is healthy twins. 


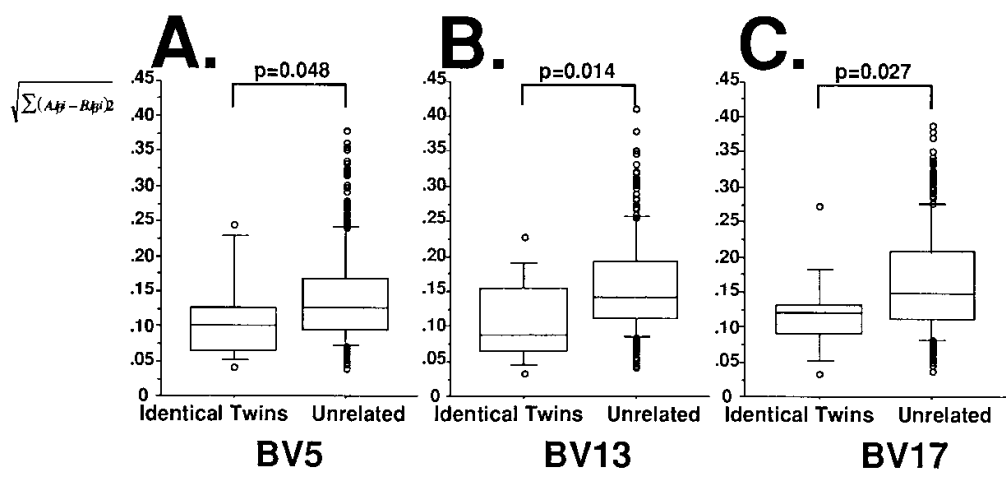

Figure 4. Comparison of TCRBJ gene repertoires of monozygotic twins and unrelated subjects. Euclidean distance (root of the sum of the squares of the differences of each combination of BJ gene repertoires) was calculated as an indicator of discordance. The whiskers contain the areas from the $10^{\text {th }}$ to $90^{\text {th }}$ percentile, boxes contain the area from the $25^{\text {th }}$ to $75^{\text {th }}$ percentile, and horizontal bars in the boxes indicate median values: $(A)$ TCRBV5, $(B)$ BV13, and (C) BV17. the determination of city-block (Manhattan) distances, using Statistica 3.0 (Biometallics Inc., Tulsa, OK).

To compare the TCRBJ gene repertoires of two subsets of donors, the Euclidean distance (square root of the sum of the squares of the difference of each BJ gene frequency) was calculated as an indicator of the discordance of the two BJ gene repertoires. $P$-values were determined with the Mann-Whitney $U$ test, using the StatView 4.0 (Abacus Concepts, Inc., Berkeley, CA).

\section{Results}

Specificity and reproducibility of the TCRBJ gene repertoire analysis. The TCRBJ locus contains six BJ1 and seven BJ2 gene segments. Oligonucleotide probes used for the TCRBJ specific oligonucleotide hybridization had to specify $3^{\prime}$ nondeletable regions of $\mathrm{BJ}$ genes. The probes were homologous (maximal homology 84\%), and their GC/AT contents were diverse. We used a 3M TMAC hybridization solution to equalize the hybridization efficiencies of the probes (11).

To test cross-hybridization of the oligonucleotide probes, we probed 13 different TCR gene clones, each of which contained a different BJ gene rearranged with a BV gene. Each probe specifically hybridized to the corresponding BJ gene, and no cross-hybridization was observed under the conditions used for the study (data not shown).

To compare labeling and hybridization efficiencies of the probes, we generated DNA which included arbitrarily selected $\mathrm{BJ} 1.1 \mathrm{p}, \mathrm{BJ} 1.2 \mathrm{p}$, and $\mathrm{BJ} 1.4 \mathrm{p}$ recognition sequences in tandem. The DNA was PCR amplified with biotinylated primers. Then, various amounts of the products were hybridized with labeled BJ specific probes in the ELISA format. The variations of the concentrations determined with different probes were less than twofold (data not shown).

In the PCR-ELISA method, TCR transcripts with specific $\mathrm{BV}$ genes were amplified with BV-specific sense and BC-specific antisense primers. The sequences of the BJ gene segments did not influence PCR priming. Thus, different BJ genes should be amplified at the same efficiency and the BJ repertoire should not depend on the number of PCR cycles. In order to confirm this prediction, we amplified the TCRBV13 transcripts from a single PBL cDNA sample with three different programs (27 cycles, 30 cycles, and 35 cycles), and determined BJ gene usage. The TCRBJ gene repertoire from three different PCR cycles showed the same BJ gene usage pattern (data not shown). The maximum disparity of the BJ gene frequencies was $4.8 \%$ and the mean difference was $2.0 \%$.

TCRBJ gene repertoires of expressed TCR with TCRBV5,
13, and 17 genes. TCRBJ gene expression, in association with TCRBV5, 13, and 17 family genes, was examined in PBL from six pairs of healthy monozygotic twins, six pairs of monozygotic twins discordant for RA, and five normal individuals (total of 29). The BV5, 13, and 17 gene families were selected, because of previous reports indicating that the frequencies of $\mathrm{T}$ cells with BV5, 13, and 17 gene products were more abundant in synovial fluid and/or PBL from RA patients $(12,13)$.

The TCRBJ gene frequencies of all 29 donors are depicted in Fig. $1 A-C$ (TCRBV5 transcripts, BV13 transcripts, and BV17 transcripts, respectively). The BJ gene usage of all three TCRBV family transcripts shared common features. In all BV family transcripts, BJ2 cluster genes were used more than $\mathrm{BJ} 1$ cluster genes, and the BJ2S1 gene was used most frequently. In contrast, the BJ1S4, BJ1S6, BJ2S4, and BJ2S6 genes were rarely used. The same usage patterns were conserved for TCRBV3, 4, 6, 14, and 15 transcripts (data not shown).

Nevertheless, as is shown in Fig. 1, each TCRBV family transcript had a distinctive BJ gene usage pattern. The TCRBV5 gene combined with the BJ2S3 gene less frequently than did other BV gene transcripts. The BV13-BJ2S1 combination was very infrequent. BV17 gene transcripts associated with the BJ1S5 gene more frequently, and used BJ1S1 and $\mathrm{BJ} 2 \mathrm{~S} 7$ genes less frequently, than predicted by chance. The other BV gene families also had distinctive BJ gene usage patterns (data not shown). In order to compare the similarity of the $\mathrm{BJ}$ repertoires for each $\mathrm{BV}$ family, a multivariate analysis was performed. The results of a cluster analysis (Ward's method and city-block [Manhattan] distances) are shown as a dendrogram in Fig. 2. The 87 different BJ gene repertoires segregated into three groups. 23 of the $29 \mathrm{BV} 5$ repertoires fell into one group, as did 27 of 29 BV13 repertoires, and 27 of 29 BV17 repertoires. The results show that the TCR transcripts from each BV gene family have a distinctive pattern of BJ gene expression in periphery.

Genetic control of TCRBJ gene repertoires. To analyze genetic effects on the TCRBJ gene repertoires of PBL, we compared the BJ gene repertoires of monozygotic twins with those of unrelated pairs. Representative results of one healthy twin pair (A1-A2) and one pair discordant for RA (G1-G2) are shown in Fig. 3. The BJ gene repertoires of the monozygotic twins were strikingly similar for all three TCRBV family transcripts. As an indicator of discordance between two given BJ gene repertoires, we used Euclidean distance (square root of the sum of the squares of the difference of each BJ gene frequency). The values for monozygotic twins were significantly smaller than those of unrelated individuals $(P<0.05)$ in all 

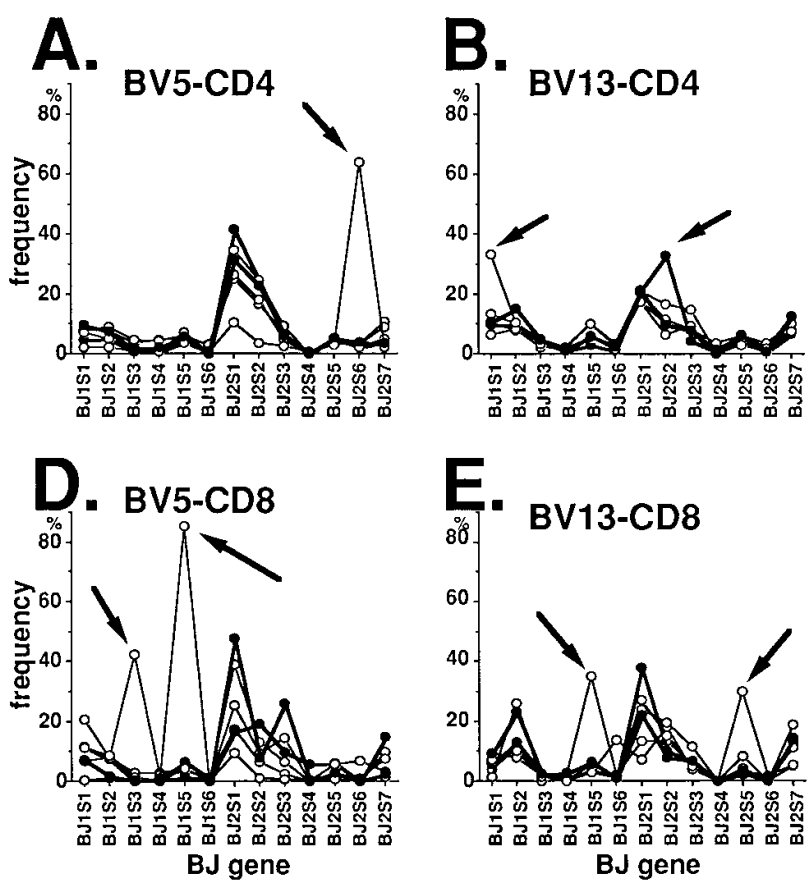

three BV families (Fig. $4 A-C$ ). Thus, the monozygotic twins had significantly more similar BJ gene repertoires than did unrelated individuals. The results show that the pattern of $\mathrm{BJ}$ gene expression in PBL is genetically controlled, and that the distribution depends on the exact genetic background.

To assess the influence of RA on peripheral TCRBJ gene repertoires, we compared the Euclidean distances between healthy twins with those between RA discordant twins. The BJ gene repertoires of PBL from RA discordant twins were statistically no more discordant than those of the healthy twins (median Euclidean distances of healthy monozygotic twins were 0.124 in TCRBV5 transcripts, 0.087 in BV13, and 0.119 in BV17; those of RA discordant monozygotic twins were 0.095 in BV5, 0.116 in BV13, and 0.124 in BV17). Furthermore, no specific changes in the BV-BJ gene combination usage were associated with RA.

TCRBJ gene repertoires of $C D 4^{+}$and $C D 8^{+} T$ cell subsets. Each TCRBV family transcript of peripheral $\mathrm{T}$ lymphocytes had a distinctive BJ gene usage pattern. To better understand the biases, we analyzed the TCRBJ gene repertoires of $\mathrm{CD}^{+}$ and $\mathrm{CD}^{+} \mathrm{T}$ cell subsets from a pair of healthy twins (B1-B2) and two pairs of RA discordant twins (G1-G2, H1- $\underline{\mathrm{H} 2})$. The $\mathrm{BJ}$ gene frequencies of three pairs of monozygotic twins (total of six) are depicted in Fig. $5 A-F$ (TCRBV5 transcripts of $\mathrm{CD}^{+}{ }^{-} \mathrm{T}$ cells, BV13 transcripts of $\mathrm{CD} 4^{+}, \mathrm{BV} 17$ transcripts of $\mathrm{CD} 4^{+}$, BV5 transcripts of $\mathrm{CD}^{+}, \mathrm{BV} 13$ transcripts of $\mathrm{CD} 8^{+}$, and BV17 transcripts of $\mathrm{CD}^{+}$, respectively). The TCRBJ gene repertoires of $\mathrm{CD}^{+} \mathrm{T}$ cells showed the same biases as those of total PBL. The BV5-BJ2S3 and BV13-BJ2S1 combinations were less frequently used, and BV17-BJ1S5 combination was more frequently used. Cluster analysis (Ward's method and city-block [Manhattan] distances) of the total of 18 different $\mathrm{BJ}$ gene repertoires of $\mathrm{CD}^{+}{ }^{+}$subsets segregated into three groups. Four of six BV5 repertoires fell into one group, as did all of six BV13 repertoires, and all of six BV17 repertoires (data not shown). On the other hand, the BJ gene repertoires of $\mathrm{CD}^{+} \mathrm{T}$ cell subsets were more divergent among
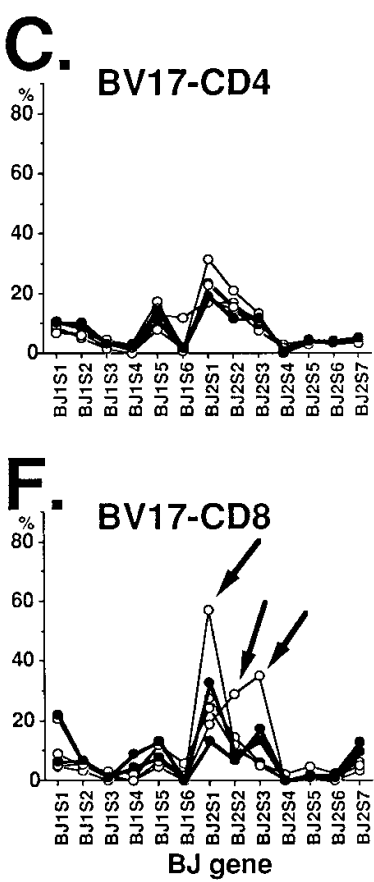

Figure 5. TCRBJ gene frequencies of $\mathrm{CD}^{+}$and $\mathrm{CD}^{+}{ }^{+} \mathrm{T}$ cell subsets of peripheral lymphocytes from a pair of healthy monozygotic twins and two pairs of monozygotic twins discordant for RA. $(A) \mathrm{BJ}$ gene repertoire of TCRBV 5 transcripts of $\mathrm{CD} 4^{+} \mathrm{T}$ cell subset. (B) BJ repertoire of BV13 transcripts of $\mathrm{CD}^{+}$. (C) BJ repertoire of BV17 transcripts of $\mathrm{CD}^{+}$. (D) BJ repertoire of BV5 transcripts of $\mathrm{CD} 8^{+}$. (E) $\mathrm{BJ}$ repertoire of BV13 transcripts of $\mathrm{CD}^{+}$. (F) BJ repertoire of BV17 transcripts of $\mathrm{CD}^{+}$. Closed circles $(\bullet)$ are RA affected, and open circles $(O)$ are healthy subjects. the twins, and were often dominated by a small number of BV-BJ combinations (depicted by arrows in Fig. 5). Multivariate analysis failed to segregate the BV-BJ groups because of the observed sporadic expansion, that was observed in both healthy

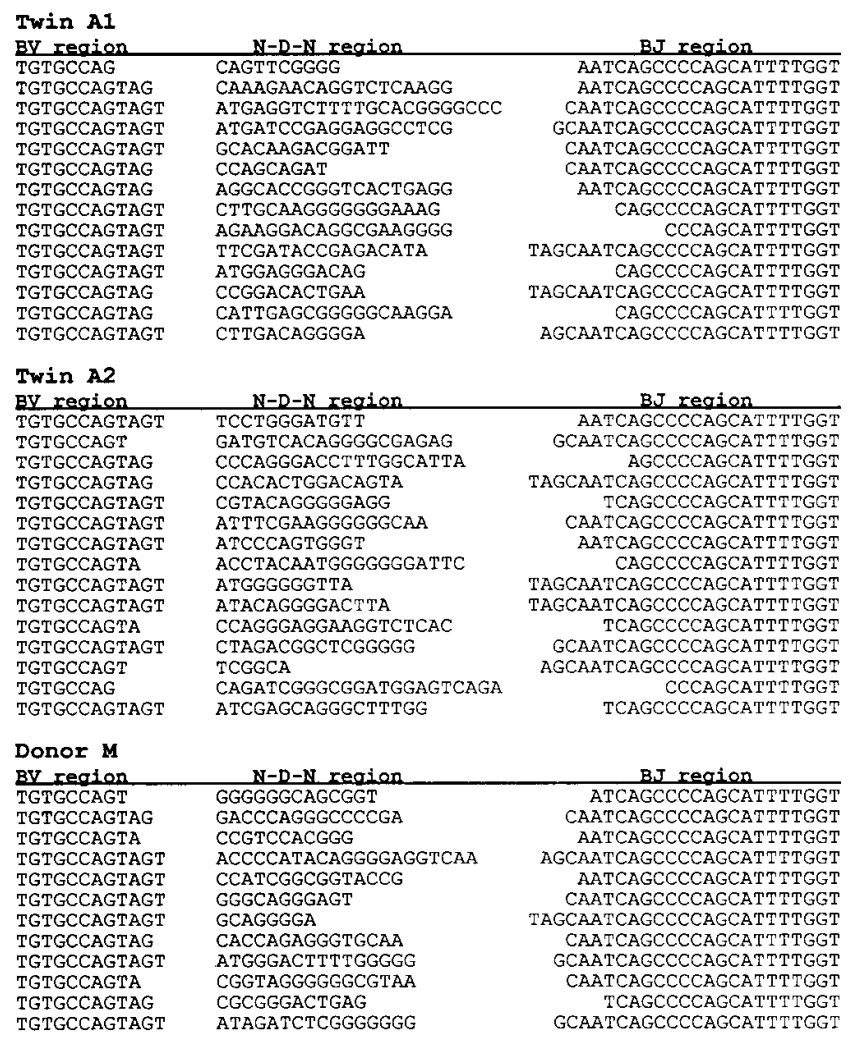

Figure 6. The junctional nucleotide sequences of TCRBV17S1J1S5 transcripts. A1 and A2 are a pair of monozygotic twins while $\mathrm{M}$ is an unrelated individual. 


\begin{tabular}{|c|c|c|}
\hline \multicolumn{3}{|c|}{ BY } \\
\hline $\mathrm{CA}$ & SSSG & NQPQHFG \\
\hline CAS & SKEQVSR & NQPQHFG \\
\hline CASS & MRSFARGP & NOPOHFG \\
\hline CASS & MIRGGLG & NQPQHFG \\
\hline CASS & AQDGF & NQPQHFG \\
\hline CAS & SQQI & NQPQHFG \\
\hline CAS & RGTGSLR & NQPQHFG \\
\hline CASS & LARGGK & QPQ $\mathrm{HFG}$ \\
\hline CASS & RRTGEGA & QHFG \\
\hline CASS & FDTETY & SNQPQHFG \\
\hline CASS & MEGQ & QPQHFG \\
\hline CAS & SRTLN & SNQPQHFG \\
\hline CAS & SIERGQG & QPQHFG \\
\hline CASS & LDRG & SNQPPQHFG \\
\hline \multicolumn{3}{|c|}{ Twin $\mathrm{A} 2$} \\
\hline BY & $\mathrm{N}-\mathrm{D}-\mathrm{N}$ & BJ region \\
\hline CASS & SWDV & NQPQHEG \\
\hline CAS & DVTGARG & NQPQHFG \\
\hline CAS & SPGTFGIK & PQHFG \\
\hline CAS & SHTGQY & SNQPQ̈HFG \\
\hline CASS & RTGGG & QPQHFG \\
\hline CASS & ISKGGN & NQPQHFG \\
\hline CASS & IPVG & NQPQHFG \\
\hline CAS & NLQWGGF & Q̊PQ̈HFG \\
\hline CASS & MGGY & SNQPQHFG \\
\hline CASS & IQGTY & SNQPPQHFG \\
\hline CAS & TREEGLT & QPQHFG \\
\hline CASS & LDGSGG & NQPPQHFG \\
\hline CAS & SA & $S N Q P Q H F G$ \\
\hline$C A$ & SRSGGWSQT & $\mathrm{QHFG}$ \\
\hline CASS & IEQGEG & QPQHFG \\
\hline \multicolumn{3}{|c|}{ Donor $M$} \\
\hline BV & $N-D-N$ & BJ region \\
\hline CAS & GGQRY & QPQHFG \\
\hline CAS & RTQGPD & NQPQHFG \\
\hline CAS & TVHG & $\mathrm{NQPQHFG}$ \\
\hline CASS & TPYRGGQ & SNQPQHFG \\
\hline CASS & PSAVP & NQPQHFG \\
\hline CASS & GQGV & NQPQHFG \\
\hline CASS & AGD & SNQPQHFG \\
\hline CAS & STRGCN & NQPQ HFG \\
\hline CASS & MGLLGG & NQPQHFG \\
\hline CAS & TVGGRN & NQPQHFG \\
\hline CAS & SAGLS & QPQHFG \\
\hline CASS & IDLGGG & $\mathrm{NQPQHFG}$ \\
\hline
\end{tabular}

Figure 7. The deduced amino acid sequences of junctional regions of TCRBV17S1J1S5 transcripts.

and RA patients. No specific BJ usage profiles in $\mathrm{CD}^{+}$or $\mathrm{CD}^{+}$population were associated with $\mathrm{RA}$.

Nucleotide and deduced amino acid sequences of the TCR $\beta$ chain junctional regions of peripheral lymphocytes from monozygotic twins. The studies described above demonstrated that the TCRBV gene and BJ gene repertoires of human peripheral $\mathrm{T}$ lymphocytes are controlled genetically. To discern any genetic effects on N-D-N sequences in the CDR3, we amplified BV-BD-BJ junctional regions of TCRBV17S1J1S5 transcripts from a pair of healthy monozygotic twins and a healthy individual. The amplicons were subcloned into plasmids for nucleotide sequencing. Preliminary experiments showed that identical clones appeared repetitively when TCR transcripts were amplified from small numbers of cells. The BV17S1J1S5 combination was selected because the BJ1S5 gene was used very frequently in BV17 gene transcripts. In ad- dition, a specific primer for BJ1S5 gene amplification was readily designed, since the BJ1S5 gene is not homologous to the consensus BJ sequence.

14 recombinant clones from $\mathrm{A} 1,15$ clones from $\mathrm{A} 2$, and 12 clones from $\mathrm{M}$ were randomly sequenced. The junctional regions are shown in Fig. 6. The average lengths of the N-D-N regions were 15.9 nucleotides in A1, 15.7 nucleotides in $\mathrm{A} 2$, and 14.1 nucleotides in $\mathrm{M}$. The N-D-N regions were similar in length and nucleotide composition in all three repertoires. TCRBD genes could not be precisely identified, because the BD1S1 and BD2S1 genes are highly homologous (14), and the $3^{\prime}$ and $5^{\prime}$ ends were truncated during rearrangement. The deduced amino acid sequences of the CDR3 are shown in Fig. 7. All sequences were unique. Hydrophobic amino acids accounted for $24.7 \%$ of the CDR3 in $\mathrm{A} 1,24.4 \%$ in $\mathrm{A} 2$, and $27.0 \%$ in M. Acidic amino acids comprised $9.9 \%$ in CDR3 in $\mathrm{A} 1,7.0 \%$ in $\mathrm{A} 2$, and $4.8 \%$ in $\mathrm{M}$. Basic amino acids covered $17.3 \%$ of the CDR3 in $\mathrm{A} 1,8.1 \%$ in $\mathrm{A} 2$, and $9.5 \%$ in M. No nucleotides or amino acid sequences or motifs of these TCR junctional regions were shared between the monozygotic twins.

\section{Discussion}

We examined the TCRBJ gene repertoires of peripheral $\mathrm{T}$ lymphocytes from 12 pairs of monozygotic twins, including 6 pairs discordant for RA, and 5 healthy individuals. The TCRBV5, 13, and 17 gene families were emphasized because of the possible association with RA. The results revealed that each BV gene family had a distinctive pattern of BJ gene usage. Moreover, the BJ gene distributions of monozygotic twins were more similar than those of unrelated individuals. Hence, the repertoire of BJ genes that form the CDR3 of the TCR must be controlled by genetic factors.

RA could be a potent environmental factor in peripheral TCR repertoire formation, because the $\mathrm{T}$ lymphocytes of affected patients express activation antigens more frequently than do normals $(15,16)$. Although all RA patients studied had active disease, the TCRBJ gene repertoires of TCRBV5, 13, and 17 transcripts of circulating $\mathrm{T}$ lymphocytes from RA discordant twins were no more different from those of healthy twins. In other experiments, we also analyzed BV3, 4, 6, 14, and 15 transcripts, which have been reported to be expanded in the synovium of subsets of RA patients $(13,17-20)$, with similar results (data not shown). Additionally, no unique changes in peripheral BJ gene expression were found in total $\mathrm{PBL}, \mathrm{CD}^{+}$, or $\mathrm{CD}^{+}$subsets from the RA patients. These results show that RA dose not induce biases in BJ usage by specific BV gene transcripts. However, because RA patients do not necessarily have altered BJ gene usage in the same BV family transcripts, disease-associated changes may not be disclosed by BJ gene repertoire analysis of populations. In this regard, we have observed that the BV repertoires of RA discordant twins vary more than those of healthy twins (Mizushima et al. unpublished data).

Our data argue that the BJ repertoire is determined primarily by genetic factors, and is not significantly altered by the chronic inflammation of RA. Previous data, which implied that RA patients had specific alterations in the peripheral TCR repertoire $(12,21-23)$, derived from analyses of heterogeneous populations, rather than from formal twin studies, as reported here. While it is still possible that HLA-dependent T 
cell repertoire formation is a major factor in RA pathogenesis (24), it is unlikely that TCR analysis alone can confirm this supposition.

Although the TCRBJ gene repertoires of monozygotic twins were more similar than those of unrelated subjects, BJ gene usage obeyed common rules in all individuals. The observation that each BV gene family had a distinct pattern of BJ usage agrees with earlier data derived from limited TCR sequence analyses (25). The genetically determined TCRBJ biases were well displayed by $\mathrm{CD} 4^{+} \mathrm{T}$ cells but poorly by $\mathrm{CD} 8^{+}$ $\mathrm{T}$ cells. Clonal expansions in peripheral $\mathrm{CD}^{+} \mathrm{T}$ cells have been reported both in healthy individuals and in autoimmune disease patients, but have not been attributed to a specific environmental stimulus (26-33). In this regard, the expanded transcripts in the RA patients were not limited to specific BV-BJ combinations.

The concordance of TCRBJ gene expression between monozygotic twins could be due to allelic polymorphisms in TCRB genes, MHC genes, and/or genes encoding self antigens involved in $\mathrm{T}$ cell education in the thymus. It is likely that the TCR conformations generated by specific BJ combinations favor surface expression of the receptor and/or optimal antigen recognition. Antigenic selection is the most powerful force driving the expansion of lymphocytes with unique TCRs. Perhaps the BJ2S1 gene product generates a TCR that reacts efficiently with antigens that are important for positive selection, or that are commonly encountered in the environment. The most common source of the peptides bound to the grooves of MHC class II molecules in the thymus are cell surface membrane proteins, including MHC molecules. Recently, Walser et al. (23) reported that the frequencies of some BV-BJ combinations correlated with HLA-DRB expression. To determine the relative roles of $\mathrm{MHC}$ and TCR genes in the selection of BJ recombinants will require analyses of siblings concordant or discordant at the MHC and TCR gene loci.

Previous studies on the TCRBJ gene repertoires of human PBL were carried out by random sequencing of TCR cDNA (25, 34-39) and by PCR-Southern blot hybridization $(40,41)$. The results of these early investigations agree with our determination that BJ2 cluster genes are more commonly used than $\mathrm{BJ} 1$ cluster genes, that the $\mathrm{BJ} 2 \mathrm{~S} 1$ gene is used very frequently and that the BJ1S4, BJ1S6, BJ2S4, and BJ2S6 genes are used uncommonly. The concordance between the data supports the conclusion that variations in probe labeling and hybridization efficiencies do not significantly interfere with TCRBJ repertoire analyses.

Differences in the recombination signal sequences of BJ genes may potentially influence the efficiency of BJ rearrangement. Indeed, it has been reported that TCRBV3S1 gene expression level depends on the allele of the spacer region in the BV3S1 gene recombination signal sequence (42). However, we compared the heptamer and nonamer sequences of each BJ gene with the consensus signal sequence (43), and found no apparent influence on the pattern of BJ gene expression.

The present studies did not disclose any genetic effects on the length, nucleotide, and amino acid composition of the N-D-N junctional regions. These results were expected, because the $\mathrm{N}$ regions are generated randomly during recombination. Whether or not RA has an effect on N-D-N sequence diversity is still unclear. However, pathogenic $\mathrm{T}$ cells from patients with systemic lupus erythematosus frequently express TCR with N-D-N regions rich in basic amino acids (44).
In summary, our analyses of the TCRBJ gene repertoires of 12 pairs of monozygotic twins and five healthy individuals by PCR-ELISA indicate that the expression of particular BJ recombinants is under genetic control. The TCRBJ repertoire remains stable despite the chronic inflammatory stimuli of RA.

\section{Acknowledgments}

The authors thank Dr. Akira Sakuma for his expert advice on the statistical analysis and Dr. Ryo Kobayashi for his assistance in the VNTR assay. We are also grateful to Nancy Noon for preparing the manuscript.

This study was supported in part by a grant-in-from the Ministry of Health and Welfare, Japan, by a grant (P01-AR-41897) from the National Institutes of Health, and by the Arthritis and Rheumatism Council, Great Britain.

\section{References}

1. WHO-IUIS Nomenclature Sub-Committee on TCR Designation. 1993. Nomenclature for T-cell receptor (TCR) gene segments of the immune system. Bull. W. H. Org. 71:113-115.

2. Wilson, R.K., E. Lai, P. Concannon, R.K. Barth, and L.E. Hood. 1988. Structure, organization and polymorphism of murine and human T-cell receptor $\alpha$ and $\beta$ chain gene families. Immunol. Rev. 101:149-172.

3. Davis, M.M. 1990. T cell receptor gene diversity and selection. Annu. Rev. Biochem. 59:475-496.

4. Kohsaka, H., A. Taniguchi, P.P. Chen, W.E. Ollier, and D.A. Carson. 1993. The expressed $\mathrm{T}$ cell receptor V gene repertoire of rheumatoid arthritis monozygotic twins: rapid analysis by anchored polymerase chain reaction and enzyme-linked immunosorbent assay. Eur. J. Immunol. 23:1895-1901.

5. Akolkar, P.N., A.B. Gulwani, R. Pergolizzi, R.D. Bigler, and J. Silver. 1993. Influence of HLA genes on T cell receptor V segment frequencies and expression levels in peripheral blood lymphocytes. J. Immunol. 150:2761-2773.

6. Davis, M.M., and P.J. Bjorkman. 1988. T-cell antigen receptor genes and T-cell recognition. Nature (Lond.). 334:395-402.

7. Claverie, J.M., C.A. Prochnicka, and L. Bougueleret. 1989. Implications of a Fab-like structure for the T-cell receptor. Immunol. Today. 10:10-14.

8. Rand, S., C. Puers, K. Skowasch, P. Wiegand, B. Budowle, and B. Brinkmann. 1992. Population genetics and forensic efficiency data of 4 AMPFLP's. Int. J. Leg. Med. 104:329-333.

9. Arnett, F.C., S.M. Edworthy, D.A. Bloch, D.J. McShane, J.F. Fries, N.S Cooper, L.A. Healey, S.R. Kaplan, M.H. Liang, H.S. Luthra et al. 1988. The American Rheumatism Association 1987 revised criteria for the classification of rheumatoid arthritis. Arthritis Rheum. 31:315-324.

10. Wucherpfennig, K.W., K. Ota, N. Endo, J.G. Seidman, A. Rosenzweig, H.L. Weiner, and D.A. Hafler. 1990. Shared human T cell receptor V $\beta$ usage to immunodominant regions of myelin basic protein. Science (Wash. DC). 248: 1016-1019.

11. Wood, W.I., J. Gitschier, L.A. Lasky, and R.M. Lawn. 1985. Base composition-independent hybridization in tetramethylammonium chloride: a method for oligonucleotide screening of highly complex gene libraries. Proc. Natl. Acad. Sci. USA. 82:1585-1588.

12. Zagon, G., J.R. Tumang, Y. Li, S.M. Friedman, and M.K. Crow. 1994 Increased frequency of V $\beta 17$-positive $\mathrm{T}$ cells in patients with rheumatoid arthritis. Arthritis Rheum. 37:1431-1440.

13. Lunardi, C., C. Marguerie, and A.K. So. 1992. An altered repertoire of $\mathrm{T}$ cell receptor $\mathrm{V}$ gene expression by rheumatoid synovial fluid $\mathrm{T}$ lymphocytes. Clin. Exp. Immunol. 90:440-446.

14. Toyonaga, B., Y. Yoshikai, V. Vadasz, B. Chin, and T.W. Mak. 1985. Organization and sequences of the diversity, joining, and constant region genes of the human T-cell receptor $\beta$ chain. Proc. Natl. Acad. Sci. USA. 82:8624-8628.

15. Cush, J.J., and P.E. Lipsky. 1988. Phenotypic analysis of synovial tissue and peripheral blood lymphocytes isolated from patients with rheumatoid arthritis. Arthritis Rheum. 31:1230-1238.

16. Smith, M.D., and T.P. Roberts. 1990. Lymphocyte surface marker expression in rheumatic diseases: evidence for prior activation of lymphocytes in vivo. Ann. Rheum. Dis. 49:81-87.

17. Paliard, X., S.G. West, J.A. Lafferty, J.R. Clements, J.W. Kappler, P. Marrack, and B.L. Kotzin. 1991. Evidence for the effects of a superantigen in rheumatoid arthritis. Science (Wash. DC). 253:325-329.

18. Howell, M.D., J.P. Diveley, K.A. Lundeen, A. Esty, S.T. Winters, D.J. Carlo, and S.W. Brostoff. 1991. Limited T-cell receptor $\beta$-chain heterogeneity among interleukin 2 receptor-positive synovial T cells suggests a role for superantigen in rheumatoid arthritis. Proc. Natl. Acad. Sci. USA. 88:10921-10925.

19. Maruyama, T., I. Saito, S. Miyake, H. Hashimoto, K. Sato, H. Yagita, K. 
Okumura, and N. Miyasaka. 1993. A possible role of two hydrophobic amino acids in antigen recognition by synovial T cells in rheumatoid arthritis. Eur. J. Immunol. 23:2059-2065.

20. Jenkins, R.N., A. Nikaein, A. Zimmermann, K. Meek, and P.E. Lipsky. 1993. T cell receptor V $\beta$ gene bias in rheumatoid arthritis. J. Clin. Invest. 92 : 2688-2701.

21. DerSimonian, H., M. Sugita, D.N. Glass, A.L. Maier, M.E. Weinblatt, T. Reme, and M.B. Brenner. 1993. Clonal V $\alpha 12.1^{+} \mathrm{T}$ cell expansions in the peripheral blood of rheumatoid arthritis patients. J. Exp. Med. 177:1623-1631.

22. Goronzy, J.J., B.P. Bartz, W. Hu, M.C. Jendro, K.D. Walser, and C.M. Weyand. 1994. Dominant clonotypes in the repertoire of peripheral CD4 ${ }^{+} \mathrm{T}$ cells in rheumatoid arthritis. J. Clin. Invest. 94:2068-2076.

23. Walser, K.D., C.M. Weyand, A.J. Weaver, W.M. O'Fallon, and J.J. Goronzy. 1995. Mechanisms underlying the formation of the T cell receptor repertoire in rheumatoid arthritis. Immunity. 2:597-605.

24. Albani, S., E. Keystone, J.L. Nelson, W. Ollier, A. Lacava, A.C. Montemayor, D.A. Weber, C. Montecucco, A. Martini, and D.A. Carson. 1995. Positive selection in autoimmunity-abnormal immune responses to a bacterial dnaj antigenic determinant in patients with early rheumatoid arthritis. Nat. Med. 1: $448-452$.

25. Quiros, E.R., A. Sottini, A. Bettinardi, A. Albertini, L. Imberti, and D. Primi. 1995. Different TCRBV genes generate biased patterns of V-D-J diversity in human T cells. Immunogenetics. 41:91-100.

26. Posnett, D.N., A. Gottlieb, J.B. Bussel, S.M. Friedman, N. Chiorazzi, Y. Li, P. Szabo, N.R. Farid, and M.A. Robinson. 1988. T cell antigen receptors in autoimmunity. J. Immunol. 141:1963-1969.

27. Grunewald, J., T.M. Jeddi, H. Dersimonian, R. Andersson, and H. Wigzell. 1992. A persistent $\mathrm{T}$ cell expansion in the peripheral blood of a normal adult male: a new clinical entity? Clin. Exp. Immunol. 89:279-284.

28. Hingorani, R., I.H. Choi, P. Akolkar, A.B. Gulwani, R. Pergolizzi, J. Silver, and P.K. Gregersen. 1993. Clonal predominance of T cell receptors within the $\mathrm{CD}^{+} \mathrm{CD}^{2} 5 \mathrm{RO}^{+}$subset in normal human subjects. J. Immunol. 151:57625769.

29. Posnett, D.N., R. Sinha, S. Kabak, and C. Russo. 1994. Clonal populations of $\mathrm{T}$ cells in normal elderly humans: the T cell equivalent to "benign monoclonal gammapathy." J. Exp. Med. 179:609-618.

30. Masuko, K., T. Kato, Y. Ikeda, M. Okubo, Y. Mizushima, K. Nishioka, and K. Yamamoto. 1994. Dynamic changes of accumulated T cell clonotypes during antigenic stimulation in vivo and in vitro. Int. Immunol. 6:1959-1966.

31. Morley, J.K., F.M. Batliwalla, R. Hingorani, and P.K. Gregersen. 1995 Oligoclonal $\mathrm{CD} 8^{+} \mathrm{T}$ cells are preferentially expanded in the $\mathrm{CD} 57^{+}$subset. $J$. Immunol. 154:6182-6190.
32. Monteiro, J., R. Hingorani, I.H. Choi, J. Silver, R. Pergolizzi, and P.K. Gregersen. 1995. Oligoclonality in the human $\mathrm{CD}^{+} \mathrm{T}$ cell repertoire in normal subjects and monozygotic twins-implications for studies of infectious and autoimmune diseases. Mol. Med. 1:614-624.

33. Fitzgerald, J.E., N.S. Ricalton, A.C. Meyer, S.G. West, H. Kaplan, C. Behrendt, and B.L. Kotzin. 1995. Analysis of clonal CD8 ${ }^{+} \mathrm{T}$ cell expansions in normal individuals and patients with rheumatoid arthritis. J. Immunol. 154: 3538-3547.

34. Toyonaga, B., and T.W. Mak. 1987. Genes of the T-cell antigen receptor in normal and malignant T cells. Annu. Rev. Immunol. 5:585-620.

35. Moss, P.A., W.M. Rosenberg, and J.I. Bell. 1992. The human T cell receptor in health and disease. Annu. Rev. Immunol. 10:71-96.

36. Rosenberg, W.M., P.A. Moss, and J.I. Bell. 1992. Variation in human T cell receptor $\mathrm{V} \beta$ and $\mathrm{J} \beta$ repertoire: analysis using anchor polymerase chain reaction. Eur. J. Immunol. 22:541-549.

37. Robinson, M.A. 1992. Usage of human T-cell receptor V $\beta, J \beta, C \beta$, and $\mathrm{V} \alpha$ gene segments is not proportional to gene number. Hum. Immunol. 35:6067.

38. Raaphorst, F.M., E.L. Kaijzel, M.J. van Tol, J.M. Vossen, and P.J. vanden Elsen. 1994. Non-random employment of V $\beta 6$ and $\mathrm{J} \beta$ gene elements and conserved amino acid usage profiles in CDR3 regions of human fetal and adult TCR $\beta$ chain rearrangements. Int. Immunol. 6:1-9.

39. Hall, M.A., and J.S. Lanchbury. 1995. Healthy human T-cell receptor $\beta$-chain repertoire-quantitative analysis and evidence for $\mathrm{J} \beta$-related effects on CDR3 structure and diversity. Hum. Immunol. 43:207-218.

40. Grunewald, J., T.M. Jeddi, E. Pisa, C.H. Janson, R. Andersson, and H. Wigzell. 1992. Analysis of $\mathrm{J} \beta$ gene segment usage by $\mathrm{CD} 4^{+}$and $\mathrm{CD} 8^{+}$human peripheral blood T lymphocytes. Int. Immunol. 4:643-650.

41. Jeddi, T.M., J. Grunewald, V. Hodara, R. Andersson, and H. Wigzell. 1994. Nonrandom T-cell receptor $\mathrm{J} \beta$ usage pattern in human $\mathrm{CD} 4^{+}$and $\mathrm{CD} 8^{+}$ peripheral T cells. Hum. Immunol. 40:93-100.

42. Posnett, D.N., C.S. Vissinga, C. Pambuccian, S. Wei, M.A. Robinson, D. Kostyu, and P. Concannon. 1994. Level of human TCRBV3S1 (Vß3) expression correlates with allelic polymorphism in the spacer region of the recombination signal sequence. J. Exp. Med. 179:1707-1711.

43. Tonegawa, S. 1983. Somatic generation of antibody diversity. Nature (Lond.). 302:575-581.

44. Desai, M.A., C. Mao, S. Rajagopalan, T. Robinson, and S.K. Datta. 1995. Structure and specificity of $T$ cell receptors expressed by potentially pathogenic anti-DNA autoantibody-inducing $\mathrm{T}$ cells in human lupus. J. Clin. Invest. 95:531-541. 